\title{
Study of Acid Phosphatase and Heamoglobin Level in Malarial and Non Malarial Fever Patients
}

\author{
Maneesh Sulya ${ }^{1}$ and Ummed Singh Solanki*2 \\ ${ }^{1}$ Department of Pathology, Gandhi Medical College, Bhopal M.P. (India) \\ ${ }^{2}$ Department of Biochemistry, Gandhi Medical College, Bhopal M.P. (India)
}

\begin{abstract}
Background: Malaria is a mosquito borne disease. It is a major health hazard in India and other tropical countries. An estimated 198 million cases of malaria and 584000 malaria deaths occurred in 2013. Acid phosphatase (ec 3.1.3.2, ACP) includes all the phosphatases with optimal activity below the $\mathrm{pH}$ of 7.0. ACP is present in lysosomes in all cells and exist extra-lysosomally in erythrocytes. Little is known about the levels of ACP in infectious diseases like malaria. So we undertook this study to know the Hb and ACP level in malarial patients.

Methods: 60 subjects in the age group of 20 to 50 years were included in this study. These subjects were divided into 2 groups. Group 1 included 30 confirmed malaria fever patients. Group 2 included 30 non-malarial fever patients of same age \& sex group. These selected subjects had no prostate problems, previous anaemia, or any other kind of bone disorder. Serum ACP was estimated using kit method

Result: The serum ACP levels were significantly increased in malarial fever patients when compared with the non malaria fever patients $(\mathrm{P}=0.032)$. There is also statistical significant difference in the Hb levels between malarial and non-malarial fever patients

Conclusion: Our results of increased levels of serum ACP and decreased $\mathrm{Hb}$ in malarial fever patients could be used as a marker for haemolysis \& anemia. ACP may be used as an additional investigation in the diagnosis of malaria.
\end{abstract}

\section{Keywords: Acid Phosphatase, Malaria, Hemoglobin}

\section{Introduction}

Malaria is a mosquito borne disease which spreads by the bite of the anopheles mosquito and rarely by blood transfusion. Four species of Plasmodium cause malaria in humans. These are P.falciparum, P.vivax, P.malariae and P.Ovale ${ }^{[1]}$. Out of these four, P.Falciparum is more responsible for mortality and severe complications, which include cerebral malaria, anaemia and renal failure ${ }^{[2]}$.

It is a major health hazard in India and other tropical countries $^{[3]}$. An estimated 198 million cases of malaria and 584000 malaria deaths occurred in $2013^{[4]}$. Under the name of acid phosphatase (ec 3.1.3.2, ACP) are included all the phosphatases with optimal activity below the $\mathrm{pH}$ of 7.0. ACP is present in lysosomes in all cells and exist extralysosomally in erythrocytes ${ }^{[5]}$. Its levels are increased in various diseases like prostate cancer that has spread to the prostate gland and to the bone, Paget's disease, hemolytic anemia, prostatitis, thrombophlebitis, Gaucher's disease, hyperparathyroidism etc. which helps in their diagnosis ${ }^{[6]}$. Lysosomal and prostatic enzymes are inhibited by tartarate ions whereas erythrocytic and bone isoenzymes are not ${ }^{[7]}$. Erythrocytic ACP gene is located on chromosome 2, osteoclast ACP is on chromosome 19 and prostatic ACP gene is on chromosome $13^{[8]}$. Little is known about the levels of ACP in infectious diseases like malaria. So we undertook this study to know the $\mathrm{Hb}$ and ACP level in malarial patients.

\section{Material and Methods}

The study group, taken from Central Pathology Lab and Medicine Department of Hamidia Hospital and Gandhi Medical College Bhopal, consisted of 60 subjects in the age group of 20 to 50 years. These subjects were divided into 2 groups. Group 1 included 30 confirmed malaria fever patients (16 P. Vivax malaria,11 P. falciparum malaria, and 3 mixed malaria). Group 2 included 30 non-malarial fever patients of same age \& sex group. These selected subjects had no prostate problems, previous anaemia, or any other kind of bone disorder.

Patients were interviewed for demographic data such as age, sex, occupation etc. were noted. Histories of similar complaints in past and current treatment were noted. Patients were subjected to a thorough physical examination, vitals (pulse rate, temperature, blood pressure and respiratory rate) and other clinical signs and symptoms suggestive of malaria infection were noted. Systemic examination was carried out.

Two specimen bottles were used for each subject. Anticoagulant bottle containing $\mathrm{K}_{2}$ EDTA for malaria 
parasite test and Heamoglobin estimation and plain bottle for acid phosphatse assay. $5 \mathrm{ml}$ blood sample were collected by clean vene-puncture from the anticubital fossa. The samples in $\mathrm{K}_{2}$ EDTA anticoagulant bottle were tested immediately for malaria parasite, after staining their thick and thin films with Giemsa stain while those samples in plain tube were allowed to clot and clotted sample centrifuged at 3000rpm for 5 min to obtain the sera. This serum was used for acid phosphatase estimation.

ACP estimation was done by kit method (AGAPPE DIAGNOSTIC Ltd) using semi-autoanalyzer (Transasia erba 5X). Acid Phosphatase catalyzes the hydrolysis of alpha-naphthylphosphate, liberating the alpha-naphthol and phosphate. The alpha-naphthol is then coupled with diazotized 2-amino-5-chlorotoluene (Fast Red TR) to form diazo dye which has a strong absorbance at $405 \mathrm{~nm}$. The increase in absorbance is directly proportional to the level of ACP in the sample. Tartrate is used as specific inhibitor of the prostatic fraction. The $\mathrm{Hb}$ estimation of $\mathrm{RBC}$ was done by cyan-methemoglobin method.

\section{Results}

This cross sectional study was conducted in the Department of Pathology and Medicine Gandhi Medical College and Hamidia Hospital Bhopal. A total of 60 adult patients presenting with clinical features suggestive of malaria fever and non-malarial fever were selected for the study.

The Statistical software SPSS 15.0 was used for the analysis of the data. Descriptive statistical analysis has been carried out in the present study. Results on continuous measurements were presented on Mean \pm SD and results on categorical measurements were presented in Number (\%). Significance is assessed at $5 \%$ level of significance. Student $t$ test (two tailed, independent) has been used to find the significance of study parameters on continuous scale between two groups. The $\mathrm{Hb}$ content and the serum levels of ACP in malaria and non-malarial fever patients are given in Table no. 1. The serum ACP levels were significantly increased in malarial fever patients when compared with the non malaria fever patients $(\mathrm{P}=0.032)$. There is also statistical significant difference in the $\mathrm{Hb}$ levels between malarial and nonmalarial fever patients.

Table 1:

\begin{tabular}{|c|c|c|c|}
\hline Parameter & Malarial fever patients & Non malarial fever patients & P value \\
\hline $\mathrm{ACP}$ & $5.75 \pm 1.51$ & $4.0 \pm 1.02$ & 0.032 \\
\hline $\mathrm{Hb}$ & $9.3 \pm 1.84$ & $11.2 \pm 1.05$ & 0.021 \\
\hline
\end{tabular}

\section{Discussion}

Phosphates are a group of enzymes that are distributed throughout most cells and body fluids they belong to the class of hydrolases. That catalyse the hydrolysis of compounds containing Acyl or phosphoryl (phosphatases) ester bonds and also compounds containing peptides amide, hemiacetal bond. Different groups of phosphomonoesterase of diagnostic importance have been identified which includes:

1. Acid phosphatase

2. Alkaline phosphatase.

The splitting of inorganic phosphate from a phosphorylated substrate is due to the presence of these enzymes (phosphatase) which is located in the cytoplasm of cell i.e.alkaline and acid phosphatases ${ }^{[9]}$.Orthophosphoric monoester phosphohydrolase is acid optimum EC. 3.1.3.2. This enzyme promotes the hydrolysis of number of orthophosphate esters, given by this generic reaction.

$$
\mathrm{R}-\mathrm{OPO}_{3}+\text { acceptor -------- } \mathrm{R}-\mathrm{OH}+\text { acceptor- } \mathrm{PO}_{3}
$$

The R may be one of a number of substrates. Presently there is no clearly define specific physiological substrate for the enzyme ${ }^{[10]}$. The enzyme contains tightly bound manganese ion in the \pm 2 or \pm 3 oxidation state, in the ratio of one manganese ion per enzyme molecule.

Acid phosphatase is found mostly in the prostate followed by the liver, red blood cells, platelets and bone. The acid phosphatase is family of an enzyme that are widespread in nature. Mystery surrounds the precise functional role of these molecular facilitators despite much research, yet paradoxically human acid phosphatase have a considerable impact as a tool for clinical investigation and intervention. Slight raised acidic phosphatase occasionally found in these osteoblastic bone disease (including Paget's disease) or cholestatic liver disease. In Gaucher and when there is the destruction of platelets and red blood cells in the case of malaria infection ${ }^{[11]}$.

Erythrocyte Acid Phosphatase(EAP) is a polymorphic enzyme in humans - there are several gene types or alleles that express the erythrocyte acid phosphatase enzyme. A person may inherit the genes for any one of several possible forms of the enzyme. Particular genotypes of EAP are linked with an inherited susceptibility to haemolytic favism, a condition where acute haemolytic anaemia 
follows the ingestion of fava beans, certain drugs, or particular infections ${ }^{[12,13]}$.

The present study shows that increased ACP activity observed in malaria fever patients was statistically significant as compared with the non-malarial fever patients. Our study also shows statistically significant decrease in heamoglobin $(\mathrm{Hb})$ levels between the malarial and non-malarial fever patients. Similar findings were also noted by D'Souza B et al, Kanwar G et al, Garba IH et al, Pratinidhi SA et al. ${ }^{[6,7,14,15]}$.

Invasion of the human erythrocytes by the malarial parasite is accompanied by a variety of biological responses in the human host and in order to survive, the plasmodium parasite brings about considerable metabolic changes in the host cell ${ }^{[16,17]}$. The malaria parasite itself generates large quantities of Reactive oxygen species (ROS) and also through its interaction with phagocytic cell system. Some of these radicals attack the plasma membranes and haemoglobin. The ROS generated in the host parasite interactions can cause several biochemical changes like lysis of erythrocytes. The alterations in the major antioxidants of the erythrocytes and the peroxide lysis of the erythrocytes may result in release of enzyme like $\mathrm{ACP}^{[18]}$. The malaria parasite uses host erythrocyte $\mathrm{Hb}$ as a major nutrient source. Since the parasite has a limited capacity to synthesize amino acids denovo or to take them up exogenously, the $\mathrm{Hb}$ is thought to be broken down to provide amino acids for its growth and maturation $^{[19,20]}$.

Thus increase in serum ACP levels in malaria patients could serve as a marker for hemolysis indicating the active stage of the disease, which may be used as an additional investigation in the diagnosis of malaria.

\section{Conclusion}

Thus our results of increased levels of serum ACP and decreased $\mathrm{Hb}$ in malarial fever patients could be used as a marker for haemolysis \& anemia. Since very few studies are done on this subject there is a further need to study to use this enzyme along with its isoenzyme as a marker in malaria in addition to the routine test involved.

\section{References}

1. Jasani JH., Sancheti SM., Gheewala BS. et al. Association of Electrolyte Disturbances $(\mathrm{Na}+, \mathrm{K}+)$ with Type and Severity of Malarial Parasitic Infection. Journal of Clinical and Diagnostic Research. 2012 (Suppl-2); 6(4): 678-681.
2. Rathod S. Study of sodium and potassium ion disturbances in malaria at Ahmedabad, Gujarat, India. International Archives of Integrated Medicine, 2014;1(1):7-11.

3. Rajkumar A., Rao S., Sundaram S. Clinical outcome in Malaria-Reiterating the role of parasitic index. Indian Journal of Clinical Practice 2012; 22(9):450-453.

4. World malaria report 2014 World Health Organization.

5. Burtis, Ashwood, Bruns. Tietz Fundamentals of Clinical Chemistry. 6th ed. In:Enzymes. Pennsylvania:Saunders An imprint of Elsevier; 2012; p. 334-335.

6. D'Souza B, • Parthasarathy R, D'Souza V. Acid Phosphatase as a Marker in Malaria. Ind J Clin Biochem 2011; 26(4): 396-399

7. Kanwar G., Yadav M., et al. Elevated serum acid phosphatase: prospective malarial marker. International Journal of Research in Applied,Natural and Social Sciences 2014; 2(9):11-14.

8. Vasudevan DM, Sreekumari S. Text book of biochemistry. 3rd ed. New Delhi: Jaypee Brothers Medical Publishers (P) Limited; 2001. p. 50-2.

9. El-Ishaq A, Omoluabi T. O. Plasma activity of tartrate resistant acid phosphatase in acute malaria infection International Journal of Life Science and Engineering. 2015; 1(4): 183-188.

10. Calibreath, D. F. (1992). Chemical Chemistry. W.B Sanders. Philadelphia U.S.A. P. 162

11. Zilva, J.F. Pannal, P.R. and Mayane, P.D.(1988).Clinical chemistry in diagnosis and treatment.5th edition. LoydLuke.London.pp.454.

12. Bottini E, Gloria-Bottinin F, Borgiani P, et al. ACP1 and human adaptability. Association with common diseases: a case-control study. Hum Genet 1995;96:629-37.

13. Bottini E, Lucarelli P, Agostino R, et al. Favism: association with erythrocyte acid phosphatase phenotype. Science 1971;171:409-11.

14. Garba, Gatsing D, Uborn G; Elevated total andisoenzyme forms of acid phosphatase in falciparum malaria. Comput Rendus Biol.2006;329(2):75-8.

15. Pratinidhi SA, Agarwal SV, Sagare AA, Gawade SH, Haribhakta SV, Hegde MV, et al. Study of serum acid phosphatase levels in children with malaria. IJHSR. 2014;4(2):73-7.

16. Prassannachandra, D'Souza V, D'Souza B. Comparative study on lipid peroxidation and antioxidants vitamins E \& $\mathrm{C}$ in falciparum and vivax malaria. Ind $\mathrm{J}$ Clin Biochem. 2006;21(2):103-106.

17. Goldberg DE, Slater AF, Cerani A, Henderson GB. Hemoglobin degradation in malaria parasite Plasmodium falciparum: anordered process in a unique 
organelle. Proc Natl Acad Sci. 1990;87(8):29312935.

18. D'Souza B, d'souzav, Swagata H,Vijayalaxmi K, Namratha A.S. Erythrocyte antioxidant enzymes and their correlation with malondialdehyde in malaria. Biochemical research 2009; 20(1): 25-27.
19. Gupta CM. Red cell membrane alterations in malaria. Ind J Biochem Biophys. 1988;25:20-4.

20. Bhabhani SD, Nilini KN. Evidence for erythrocyte lipid peroxidation in acute falciparum malaria. Trans R Soc Trop Med Hyg.1999;93:58-62

*Corresponding author:

Dr. Ummed Singh Solanki, Assistant Professor, Department of Biochemistry, Gandhi Medical College, Bhopal M.P. - India

Phone: +91 9826609403

Email: ummedsolanki25@gmail.com

Financial or other Competing Interests: None. 